\title{
A visual model of the Lorentz triangular moduli space
}

\section{Daniel de la Fuente, Rafael Ramírez-Uclés, Juan Francisco Ruiz}

\begin{abstract}
We present a schematic construction of the triangular shapes space in the Lorentz plane, i.e., the moduli space of all Lorentzian triangles up to similarity.
\end{abstract}

1. INTRODUCTION Lorentzian Geometry has been intensely studied for many mathematicians since its irruption in the past century. It resulted to be very successful in Physics to model the geometry of relativistic spacetimes and, nowadays, it is a consolidated branch of Mathematics.

In particular, the Lorentz plane $\mathbb{L}^{2}$ (also named Lorentz-Minkowski plane) plays a crucial role in Special Relativity. It consists in a real affine plane endowed with the indefinite metric $\langle\rangle=,-d t^{2}+d x^{2}$, expressed in an fixed affine coordinate system $(x, t)$. A vector $v$ will be called spacelike, timelike or lightlike if its square modulus $|v|^{2}=\langle v, v\rangle$ is positive, negative or null respectively. We will denote by $\|v\|$ to the non-negative length $\sqrt{|\langle v v\rangle|}$.

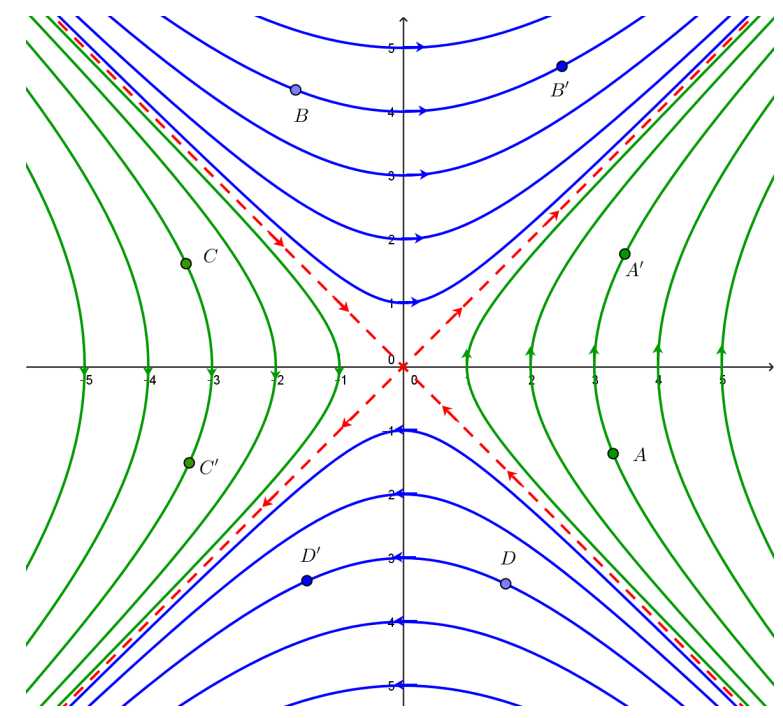

Figure 1. Lorentzian circumferences $\left(P \in \mathbb{L}^{2}:|O P|^{2}= \pm r^{2}\right)$ with positive (green) and negative (blue) radius. Arrows point in the direction in which points are moved when a boost with positive angle is applied. Points $A^{\prime}, B^{\prime}, C^{\prime}, D^{\prime}$ are the images of points $A, B, C, D$ under a boost of angle $\phi=1$.

Triangles in the Lorentz plane, as in the Euclidean case, are defined by means of three non collinear points. According to the causal character of its sides, a triangle is said to be timelike or hyperbolic (resp. spacelike) if all of its sides are timelike (resp. spacelike) and lightlike if at least one of its sides has null length. In next sections we will visualize the complete classification of triangles attending to the causal character of sides. A detailed analysis of timelike triangles is dealt in [1]. 
Our main objective here consists in to visualize the whole space of triangles under the similarity equivalence relation. Two triangles are said to be similar when have the same shape, i.e., when there exists an isometry and a homothecia of $\mathbb{L}^{2}$ carrying one triangle to the other one. Thus, we pretend to built the quotient space of all triangles in $\mathbb{L}^{2}$ by the isometry group as well as homothetic transformations. The analogous moduli space for Euclidean triangles is studied, for instance, in [3].

Recall that the isometry group of the Lorentz plane is generated by translations, symmetries respect to vertical and horizontal lines $(x=a, t=b, a, b \in \mathbb{R})$ and 'boosts' (the rotations in $\mathbb{L}^{2}$ ). A boost of hyperbolic angle $\phi \in \mathbb{R}$, represented in Fig.1, has the expression

$$
(x, t) \longmapsto(\cosh (\phi) x+\operatorname{senh}(\phi) t, \operatorname{senh}(\phi) x+\cosh (\phi) t) .
$$

In contrast to the Euclidean case, given two vectors with the same origin and modulus, it is not possible in general to transform one into the other through a boost. However, every timelike vector (resp. spacelike vector) may be carried to axis $t$ (resp. axis $X$ ) by means of a boost.

2. TRIANGULAR SHAPES SPACE AS A SET Let $A B C$ be a triangle in the Lorentz plane so that the shortest side is $\overline{A B}$. If its length is $\|A B\|=L$, we apply a homothecia centered at $A$ of ratio $1 / L$. Then, through a translation, we can place vertex $A$ at the origin $(0,0)$. Now, one of the following three cases happen.

(a) Side $\overline{A B}$ is spacelike. In this case, by means of a boost with respect to $A$, we can place vertex $B$ at the point $(1,0)$. In addition, if necessary, vertex $C$ can be located in the first quadrant by a reflectional symmetry across the axis $X$. Finally, by means of a second reflection (if necessary) across the line $x=1 / 2$, we are able to place the third vertex $C$ in the half plane $x \geq 1 / 2$.

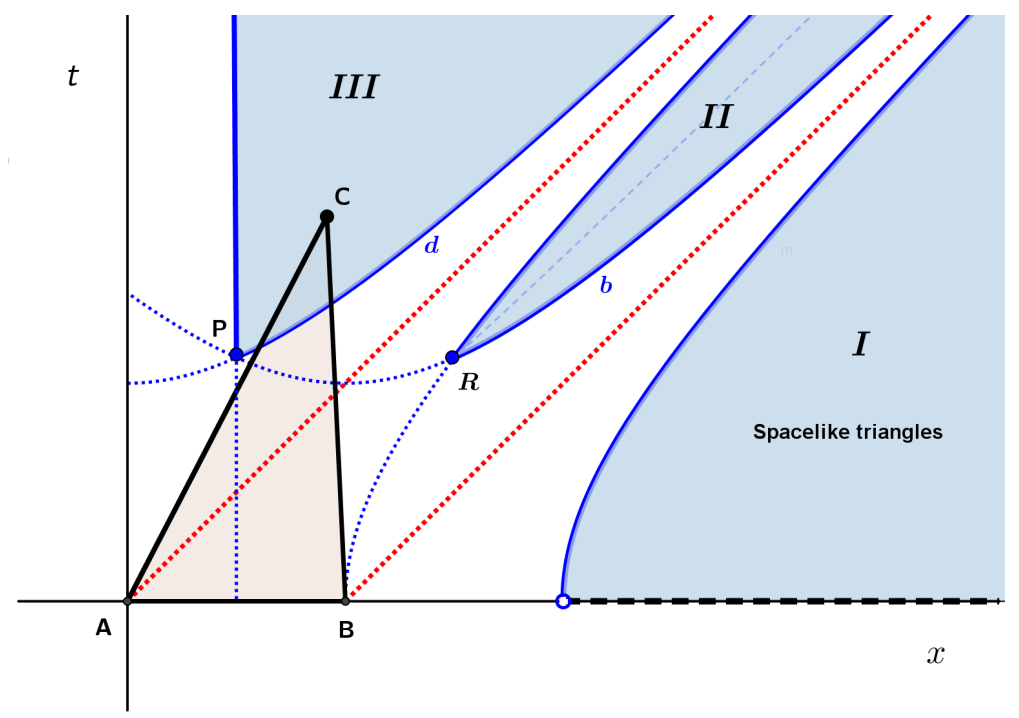

Figure 2. Each triangle with spacelike shortest side is univocally identified with a point of the blue region. Its boundary represents isosceles triangles, and points $P$ and $R$ are equilateral triangles.

Since the side $\overline{A B}$ is the shortest one, lengths $\|A C\|$ and $\|B C\|$ must be bigger than 1 . Therefore each triangle of this case may be identified with a point $C(x, t)$ 
placed in the striped area in Fig.2. We distinguish three regions, denoted by $I, I I$ and $I I I$ in Fig.2. Region $I$ corresponds with the set of spacelike triangles. In region $I I$ are located triangles with a timelike side and two spacelike sides and, in region $I I I$, triangles with two timelike sides and one spacelike. Point $R$ in the picture represents the equilateral triangle with two spacelike sides and one timelike. Similarly, $P$ is identified with the equilateral triangle with two timelike sides and one spacelike. In addition, the boundary of regions $I, I I$ and $I I I$ represents isosceles triangles with longer uneven side. For instance, boundary of $I$ are the isosceles spacelike triangles. Points of dotted line dividing $I I$ are isosceles triangles with shorter uneven side.

(b) Side $\overline{A B}$ is timelike. Then, vertex $B$, which is denoted by $B^{\prime}$ in Fig.3, may be placed at $(0,1)$ through a certain boost. If necessary, vertex $C$ can be located in the second quadrant by a reflection across the axis $X$. Finally, by means of a second reflection (if necessary) across the line $t=1 / 2$, we are able to place vertex $C$ in the half plane $t \geq 1 / 2$.

As before, since $\overline{A B^{\prime}}$ is the shortest side, then $\|A C\|,\|B C\| \geq 1$. Each equivalence class of triangles of this type is determined by point of the green area of Fig.3. There are three regions again, and its interpretation is analogous to the previous case. Note that boundaries $b$ and $d$ of $V$ and $V I$ (discontinuous green line in the picture of Fig.3) represent the same triangles of some boundaries of regions $I I$ and III.

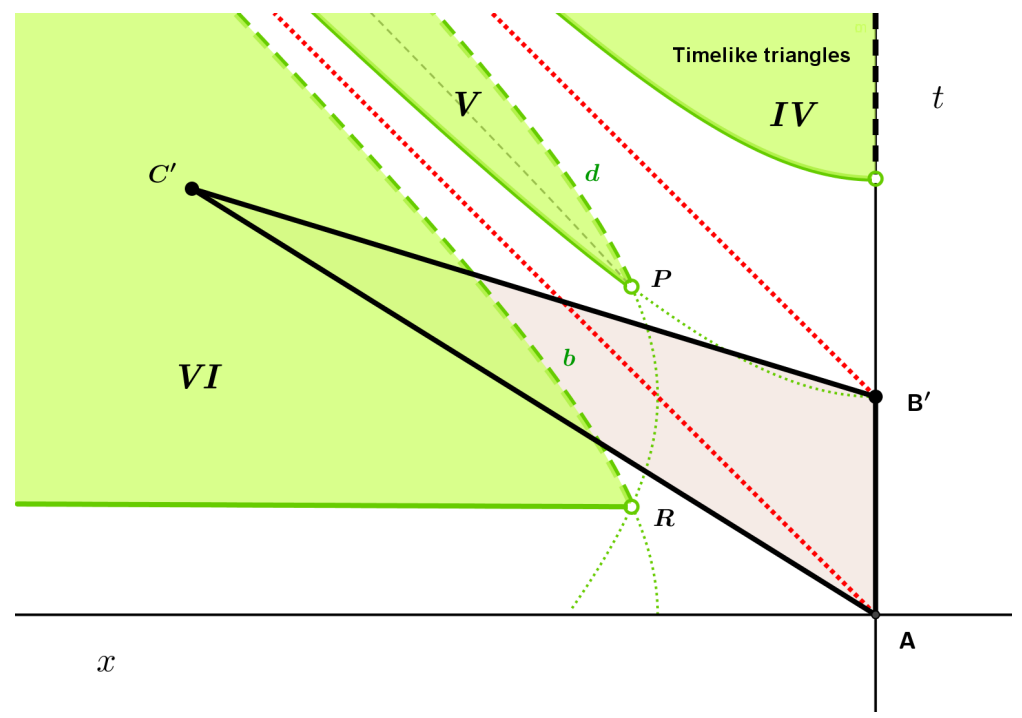

Figure 3. Each point of the green area (regions $I V, V, V I)$ univocally determines a class of triangles with timelike shortest side. Its boundary corresponds with isosceles triangles with longer uneven side. Discontinuous line dividing $V$ contains isosceles triangles with shorter uneven side.

(c) Side $\overline{A B}$ is lightlike. In this case, we have $\|A B\|=0$. Hence, between the other two sides, we choose the shortest one, which will be renamed $\overline{A B}$ if is spacelike, or $\overline{A B^{\prime}}$ if is timelike. We put vertex $A$ at the origin. The third vertex must be in one of the four semi lines drawn in Fig.4, or at the points $Q$ or $S$. The last ones represent triangles with two lightlike sides and one timelike or spacelike respectively. Point $T$ determines the class of isosceles triangles with a lightlike side. 


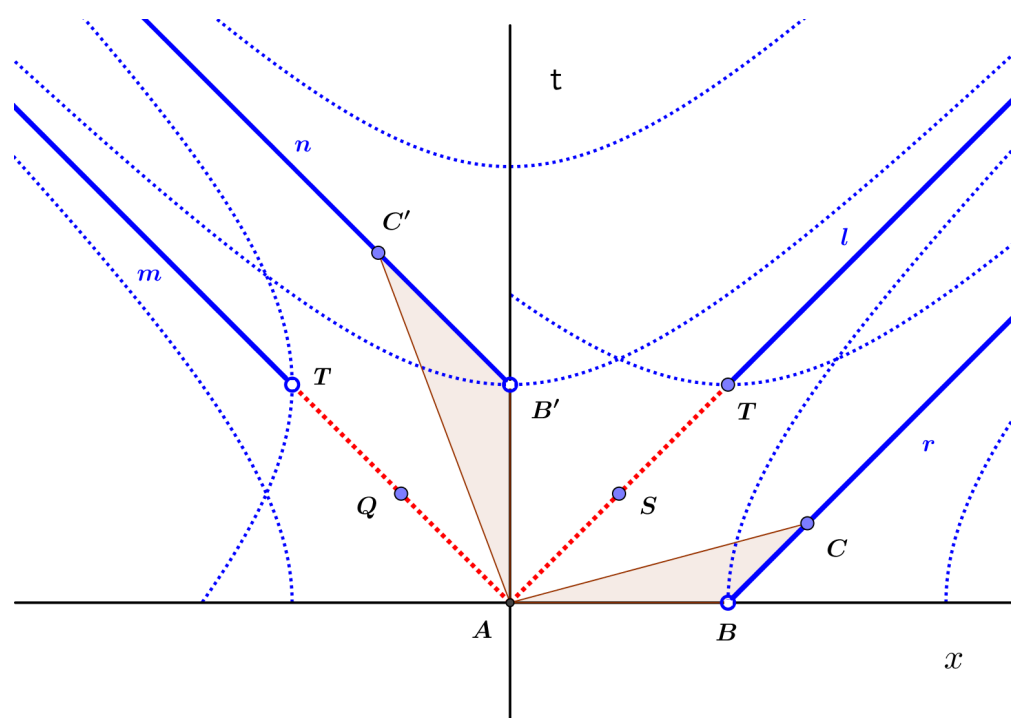

Figure 4. Lightlike triangles are represented by points in semi lines $l, m, n, r$ and points $Q$ and $S$.

We will denote the built space by $\mathcal{T}$. As an immediate consequence, this construction allow us to observe that set of lightlike triangles is much smaller than set of spacelike ones (for instance, Hausdorff dimension of lightlike triangles is one while dimension of the spacelike or timelike ones is two). Analogously, equilateral triangles have null measure while the isosceles ones have dimension one. We may also observe that there are no equilateral spacelike or timelike triangles, and it is not possible a triangle with three lightlike sides.

3. TOPOLOGY OF TRIANGULAR SHAPES SPACE In the previous section we have described the moduli space of Lorentzian triangles (up to similarity) as a set: each point of this space univocally corresponds with a triangular shape in the Lorentz plane. However, the built space cannot inherit directly the topology of $\mathbb{R}^{2}$. For instance, some points of region $I I I$ are identified with the boundary of $V$. Moreover, since an arbitrary triangle may be continuously deformed to any other one, the resulting quotient space must be connected by arcs. Now we will provide a suitable topology to our previously constructed space which allow us correctly visualize any continuous deformation of a triangle.

By one hand, observe that the equivalence class determined by a triangle $A B C$ with vertex $C$ located near $S$ along dotted red line in Fig.4, is represented by a far point of semi line $r$ or $m$. Then, $S$ links $m$ and $r$ at "infinity". Similarly, $l$ and $n$ are linked by means of $Q$, and $m$ and $l$ through point $T$. Therefore, the space of lightlike triangles actually forms an open segment with extremes $B$ and $B^{\prime}$.

In order to analyze this situation, it is convenient to make the following conformal transformation from the Lorentz half plane to $\Gamma$, usually known as Penrose-Carter map [2] (see Fig.5a),

$(x, t) \in \mathbb{L}^{2} \longmapsto(\arctan (t+x)-\arctan (t-x), \arctan (t+x)+\arctan (t-x))$,

where $\Gamma=\left\{(X, T) \in \mathbb{R}^{2}:-\pi<T+X<\pi,-\pi<T-X<\pi, T>0\right\}$.

In $\Gamma$ we may visualize the 'infinity points' of $\mathbb{L}^{2} \cap\{t>0\}$-usually named future lightlike infinity- as points of its boundary (red lines in Fig.5a). We can suitably place 
(a)

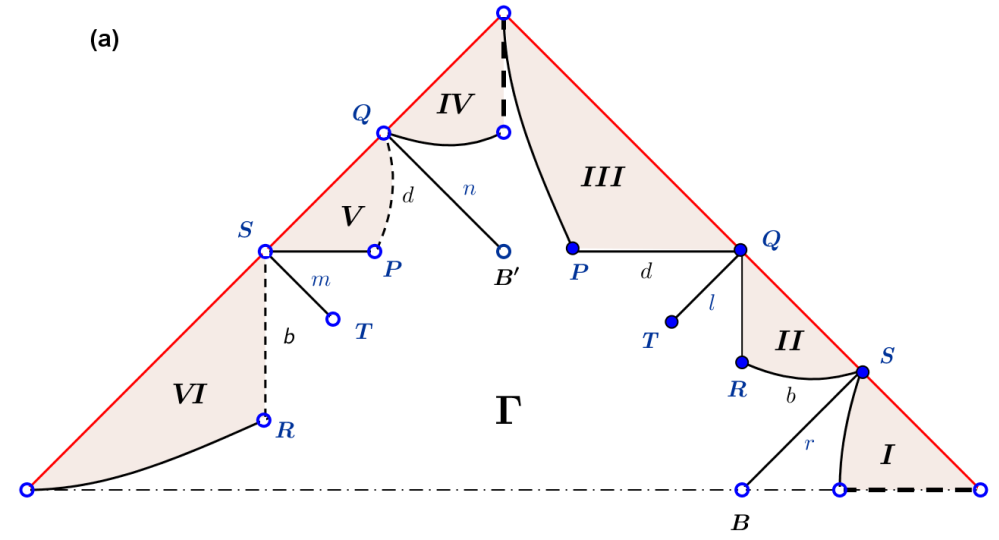

(b)

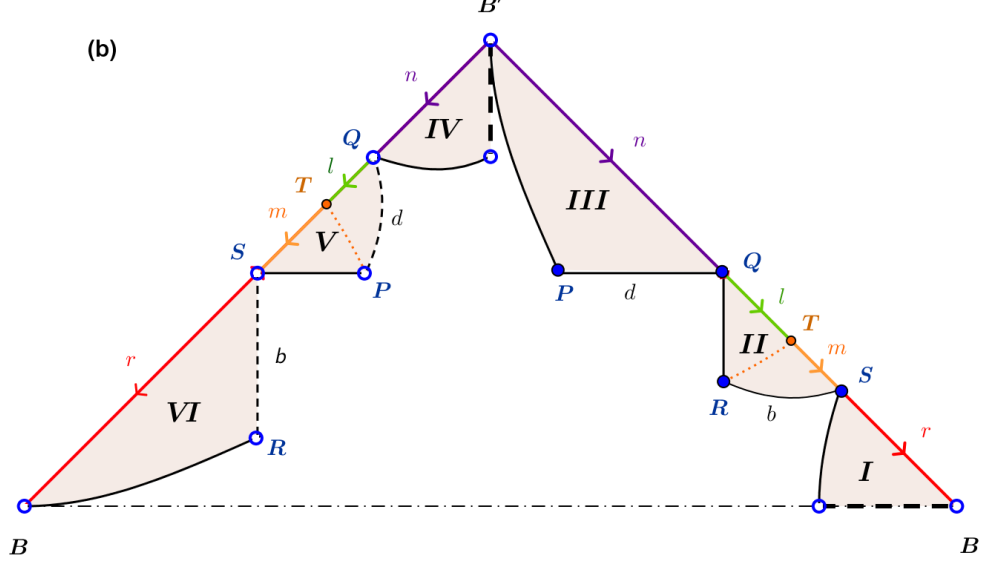

Figure 5. (a) The image of set $\mathcal{T}$ through the Penrose-Carter map. (b) Segments corresponding to classes of lightlike triangles must be removed to the boundary of $\Gamma$. Boundary segments with same colour are identified. Orange dotted lines represent classes of isosceles triangles with shorter uneven side.

points $Q$ and $S$ on it, but the resulting space is still unsatisfactory if we endow it with the topology inherited via the Penrose-Carter map. For instance, the only way to deform a spacelike triangle into another in region $I I$ would be passing through a triangle with two lightlike sides (point $S$ ), but that is not true.

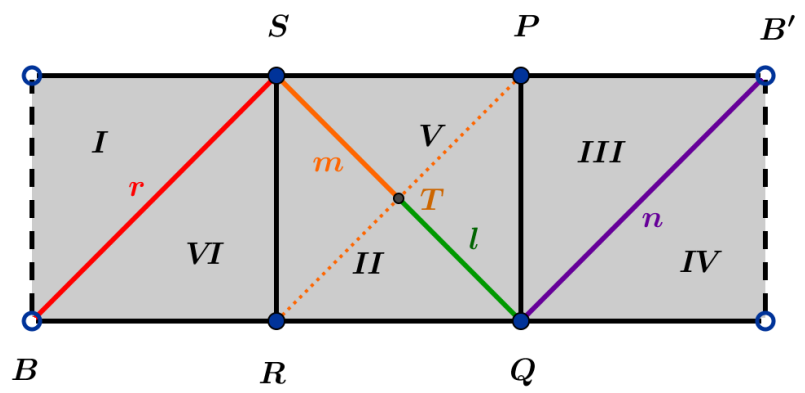

Figure 6. Triangular moduli space is topologically a band in $\mathbb{R}^{2}$. On it we may locate the different kinds of Lorentzian triangles.

Consider a continuous curve in picture of Fig.2 from a triangle $A B C$ in region $I$ 
to another one in region $I I$. Observe that the induced curve in the quotient space goes towards the 'infinity' of region $I$, passes through a class of $r$ and finally continues across region $I I$. Consequently, segment $r$ must be the 'infinity of $I$ ', as it is shown in Fig.5b. A similar detailed analysis of the 'infinity' of each region lead us to the complete space exposed in Fig.5b. The topology of this new space is the inherited from $\Gamma$ taking into account the identifications described by colours and arrows in Fig.5b. A simple homeomorphism leads us to the final triangular moduli space of Fig.6.

As summary, we conclude this article locating each type of Lorentzian triangles on picture of Fig.6.

- Regions: $I$, spacelike triangles; $I I$, two spacelike sides, one timelike with shortest spacelike side; $I I I$, two timelike sides and one shorter spacelike side; $I V$, timelike triangles; $V$, two timelike sides, one spacelike side with timelike shortest side; $V I$, two spacelike sides and a shortest timelike side.

- Lines: isosceles triangles with longest uneven side (black); isosceles with shortest uneven side (dotted orange line); $r$, lightlike triangles with two spacelike sides; $l$ and $m$, lightlike triangles with one spacelike and one timelike, in $l$ the spacelike side is shorter than the timelike one, and in $m$ the timelike side is shorter; $n$, lightlike triangles with two timelike sides.

- Points: $P$, equilateral triangle with two timelike sides and one spacelike; $R$, equilateral triangle with two spacelike sides and one timelike; $S$, two lightlike sides and one spacelike side; $Q$, two lightlike sides and one timelike side; $T$ isosceles lightlike triangle (with a timelike side and a spacelike one with the same length).

ACKNOWLEDGMENT. This work is partially supported by Spanish MINECO and ERDF project MTM201347828-C2-2-P as well as "FQM-193, Pensamiento Numérico".

\section{REFERENCES}

1. Graciela S. Birman and Katsumi Nomizu , Trigonometry in Lorentzian Geometry, Amer. Math. Monthly 91 (1984) 543-549.

2. Roger Penrose, The Light Cone at Infinity, Proceedings of the 1962 Conference on Relativistic Theories of Gravitation, Polish Academy of Sciences, Warsaw, 1965.

3. Ian Stewart, Why do all triangles form a triangle?, Amer. Math. Monthly 124 (2017) 70-73.

DANIEL DE LA FUENTE (delafuente@ugr.es) is currently a post-doctoral researcher in Department of Geometry and Topology of University of Granada (Spain). He obtained his PhD degree in Mathematics in 2016, and he is graduated in Mathematics and Physics by the University of Valladolid (Spain). He teaches graduate courses of Geometry and does research in both geometrical analysis and mathematical physics.

Departamento de Geometría y Topología, Facultad de Ciencias, C.P. 18071, Granada (Spain).

RAFAEL RAMÍREZ-UCLÉS (rramirez@ugr.es) is Assistant Professor in Department of Didactics of Mathematics at the University of Granada (Spain). He is experienced in teaching secondary school maths as well as in Degree of Primary Education and Master of Teacher Training. He completed his doctoral thesis on visualization skills of students with mathematical talent. His main line of research is attention to the high mathematical ability and the development of the spatial sense in students.

Departamento de Didáctica de la Matemática, Campus de Cartuja, C.P. 18071, Granada (Spain).

JUAN FRANCISCO RUIZ (jfruiz@ugr.es) is Assistant Professor in Department of Didactics of Mathematics at University of Granada (Spain). His career includes experience as teacher in secondary education as well as high education. He has published in partial differential equations and currently he is interested in both advanced mathematical thinking and meanings of school mathematics concepts. 
Departamento de Didáctica de la Matemática, Campus de Cartuja, C.P. 18071, Granada (Spain). 